\title{
A ventriculotomia apical esquerda para tratamento cirúrgico da estenose mitral congênita
}

\author{
Miguel BARBERO-MARCIAL**, Arlindo RISO**, Ângelo ALBUQUERQUE*, Edmar ATIK*, \\ Adib D. JATENE*
}

\begin{abstract}
BARBERO-MARCIAL, M.; RISO, A.; ALBUQUERQUE, A.; ATIK, E.; JATENE, A. D. - A ventriculotomia apical esquerda para tratamento cirúrgico da estenose mitral congênita. Rev. Bras. Cir. Cardiovasc., 6 (3):167-173, 1991.

RESUMO: Entre junho de 1987 e outubro de 1990, nove pacientes consecutivos, portadores de estenose mitral congênita (EMC) foram submetidos a correçäo cirúrgica. Sete tinham valva mitral em paráquedas e dois, outras formas complexas de estenoses. Em todos, a via de abordagem foi a ventriculotomia apical esquerda, sendo dividido, primeiramente, o músculo papilar; depois, as cordas e, finalmente, as cúspides. As lesöes associadas foram corrigidas prévia ou simultaneamente. Todos os pacientes tiveram boa evolução imediata. i Houve um óbito tardio nāo relacionado. O estudo ecocardiográfico seriado pósoperatório mostrou adequada função ventricular esquerda. Conclui-se que esta via é de escolha para tratar lesōes estenóticas congênitas complexas da valva mitral.
\end{abstract}

DESCRITORES:valva mitral, cirurgia; ventriculotomia apical esquerda.

\section{INTRODUÇĀO}

As anomalias congênitas da valva mitral ocorrem em $0,6 \%$ das necrosias de pacientes com doença congênita cardíaca e em $0,21 \%$ a $0,42 \%$ das publicaçöes clínicas de cardiopatias congênitas ${ }^{3}$.

Dentro do amplo espectro destas anomalias, a valva mitral em pára-quedas tem uma incidência significante ${ }^{6,9}$ e sua correçäo é considerada um desafio cirúrgico ${ }^{1,6}$.

Mesmo após a comissurotomia, a análise do músculo papilar único através do átrio esquerdo é difícil, por causa da exposição e pelo arranjo muscular complexo ${ }^{6}$; este problema se agrava em crianças pequenas.

Baseados nestas premissas, decidimos abor- dar a malformação principal, isto é, o aparelho subvalvar, através da ventriculotomia esquerda, o que permite um acesso completo do aparelho valvar mitral.

\section{CASUÍSTICA E MÉTODOS}

Entre junho de 1987 e outubro de 1990, nove pacientes consecutivos, com estenose mitral congênita, sendo sete por valva em pára-quedas e dois por outros tipos de estenose mitral, foram submetidos a correção cirúrgica. Todos os pacientes tinham anomalias associadas. A idade variou de três a 74 meses, sendo seis pacientes menores de 22 meses; cinco eram do sexo masculino e quatro do sexo feminino (Tabela 1).

Trabalho realizado no Instituto do Coração do Hospital das Clínicas da Faculdade de Medicina da Universidade de São Paulo e no Hospital SírioLibanês. São Paulo, SP, Brasil.

Apresentado ao $18^{\circ}$ Congresso Nacional de Cirurgia Cardíaca. Rio de Janeiro, RJ, 5 e 6 de abril, 1991.

* Do Instituto do Coração do Hospital das Clínicas da Faculdade de Medicina da Universidade de São Paulo e do Hospital Sírio Libanês.

* Do Instituto do Coração do Hospital das Clínicas da Faculdade de Medicina da Universidade de São Paulo.

Endereço para separatas: Miguel Barbero-Marcial. Av. Dr. Eneas Carvalho de Aguiar, 44. Divisão Cirúrgica. 05403 São Paulo, SP, Brasil. 
BARBERO-MARCIAL, M.; RISO, A.; ALBUQUERQUE, A.; ATIK, E.; JATENE, A. D. - A ventriculotomia apical esquerda para tratamento cirúrgico da estenose mitral congênita. Rev. Bras. Cir. Cardiovasc., 6 (3):167-173, 1991.

TABELA 1

ANOMALIAS ASSOCIADAS E TÉCNICA OPERATÓRIA EM PACIENTES COM ESTENOSE MITRAL CONGÊNITA

\begin{tabular}{|c|c|c|c|c|c|}
\hline $\begin{array}{l}\text { Paciente } \\
\quad N^{\circ}\end{array}$ & $\begin{array}{c}\text { Idade } \\
\text { (meses) } \\
\text { Sexo }\end{array}$ & $\begin{array}{l}\text { Anomalias } \\
\text { Associadas }\end{array}$ & $\begin{array}{c}\text { Operações } \\
\text { Prévias }\end{array}$ & $\begin{array}{l}\text { Procedimento } \\
\text { Cirúrgico }\end{array}$ & $\begin{array}{c}\text { Periodo de Pós-Operatório } \\
\text { (meses)e Classe Funcional } \\
\text { (NYHA) }\end{array}$ \\
\hline 1 & $22, F$ & $\begin{array}{l}\text { CIV's múltiplas } \\
\text { PCA }\end{array}$ & - & $\begin{array}{l}\text { Abertura aparelho } \\
\text { mitral. Fechamento de } \\
\text { CIV's múltiplas e ductus }\end{array}$ & $44-1$ \\
\hline 2 & 18, M & $\begin{array}{l}\text { Coarctação de aorta } \\
\text { PCA. Membrana supra- } \\
\text { valvar }\end{array}$ & $\begin{array}{l}\text { Istmosubclavio- } \\
\text { plastia } \\
\text { Fechamento do } \\
\text { ductus }\end{array}$ & $\begin{array}{l}\text { Abertura aparelho mitral } \\
\text { Ressecção de membrana } \\
\text { supravalvar }\end{array}$ & $20-1$ \\
\hline 3 & 3, M & $\begin{array}{l}\text { Coarctação de aorta } \\
\text { CIV's múltiplas }\end{array}$ & - & $\begin{array}{c}\text { Abertura aparelho } \\
\text { valvar - fechamento de } \\
\text { CIV's } \\
\text { Istmosubclavioplastia }\end{array}$ & $18-1$ \\
\hline 4 & $5, F$ & $\begin{array}{c}\text { Coarctação de aorta } \\
\text { PCA }\end{array}$ & $\begin{array}{l}\text { Istmosubclavio- } \\
\text { plastia } \\
\text { Fechamento do } \\
\text { ductus }\end{array}$ & $\begin{array}{c}\text { Abertura aparelho } \\
\text { mitral }\end{array}$ & $17-1$ \\
\hline 5 & $74, M$ & $\begin{array}{c}\text { DVSVD C/ CIV } \\
\text { Subaórtica } \\
\text { PCA }\end{array}$ & $\begin{array}{l}\text { Correção da } \\
\text { DVSVD } \\
\text { Fechamento do } \\
\text { ductus }\end{array}$ & $\begin{array}{l}\text { Abertura aparelho } \\
\text { mitral } \\
\text { Ressecção estenose } \\
\text { subaórtica }\end{array}$ & $16-1$ \\
\hline 6 & $8, M$ & $\begin{array}{l}\text { DVSVD com CIV } \\
\text { restritiva e não } \\
\text { relacionada. PCA }\end{array}$ & $\begin{array}{l}\text { Cerclagem } \\
\text { pulmona } \\
\text { Fechamento do } \\
\text { ductus }\end{array}$ & $\begin{array}{l}\text { Abertura aparelho } \\
\text { mitral } \\
\text { Aumento da CIV } \\
\text { Septectomia atrial }\end{array}$ & $15-I 1$ \\
\hline 7 & $19, \mathrm{~F}$ & Duplo aparelho mitral & - & $\begin{array}{l}\text { Abertura dos dois } \\
\text { aparelhos mitrais } \\
\text { Fechamento de } \mathrm{CIV} \\
\text { e canal }\end{array}$ & $15-1$ \\
\hline 8 & $14, M$ & Estenose subaórtica & - & $\begin{array}{l}\text { Abertura aparelho } \\
\text { mitral. Ressecção } \\
\text { est. subaórtica }\end{array}$ & $14-1$ \\
\hline 9 & $10, F$ & Coarctação da aorta & - & $\begin{array}{l}\text { Abertura aparelho } \\
\text { mitral. Ressecção da } \\
\text { CoAo e anastomose }\end{array}$ & $6-1$ \\
\hline
\end{tabular}

A classe funcional foi IV em quatro pacientes (insuficiência cardíaca, dispnéia, taquicardia e hepatomegalia) e classe funcional III em cinco pacientes (episódios freqüentes de infecção pulmonar ou insuficiência cardíaca com hipodesenvolvimento pondoestatural).

O estudo ecocardiográfico demonstrou o tipo da estenose mitral e as anomalias associadas em todos os pacientes. Entretanto, em três pacientes ecocardiogramas prévios não tinham demonstrado a anomalia mitral. Quatro pacientes foram submetidos a estudo hemodinâmico e apresentavam pressōes sistêmicas em tronco pulmonar (TP) com gradientes transvalvares miltrais significantes. As lesōes associadas encontram-se enumeradas na Tabela 1.

Cinco pacientes foram submetidos a operaçōes prévias, três a istmosubclavioplastias, um cerclagem do tronco pulmonar e uma correção da dupla via de saída do ventrículo direito com comunicação interventricular subaórtica. Neste último doente, o diagnóstico de estenose mitral e subaórtica foi feito três anos após o procedimento inicial.

Todos os pacientes foram operados através de 
BARBERO-MARCIAL, M.; RISO, A.; ALBUQUERQUE, A.; ATIK, E.; JATENE, A. D. - A ventriculotomia apical esquerda para tratamento cirúrgico da estenose mitral congênita. Rev. Bras. Cir. Cardiovasc., 6 (3):167-173, 1991.

esternotomia mediana com o auxílio da circulação extracorpórea e hipotermia profunda. Após o clampeamento aórtico e a infusão de cardioplegia, a valva mitral foi explorada através do átrio esquerdo em três pacientes e do septo interatrial em dois. Nos restantes quatro pacientes, a inspeção inicial da valva mitral foi feita através de ventriculotomia esquerda apical. A correção da malformação foi realizada através do ventrículo esquerdo em todos os casos. A ventriculotomia no ápex do ventrículo esquerdo foi feita a $5 \mathrm{~mm}$ da artéria interventricular anterior, prolongando-a para a face anterior e posterior, tendo o comprimento de aproximadamente $2 \mathrm{~cm}$.

Através da incisão, o aparelho subvalvar foi cuidadosamente avaliado. O músculo papilar único foi dividido em duas partes, lateral e medial; $a$ incisão penetrou cerca de $2 \mathrm{~mm}$ na espessura da parede ventricular, para possibilitar maior mobilidade. Os músculos resultantes foram "debastados" para tornálos menos obstrutivos ao fluxo sangüíneo (Figura 1).

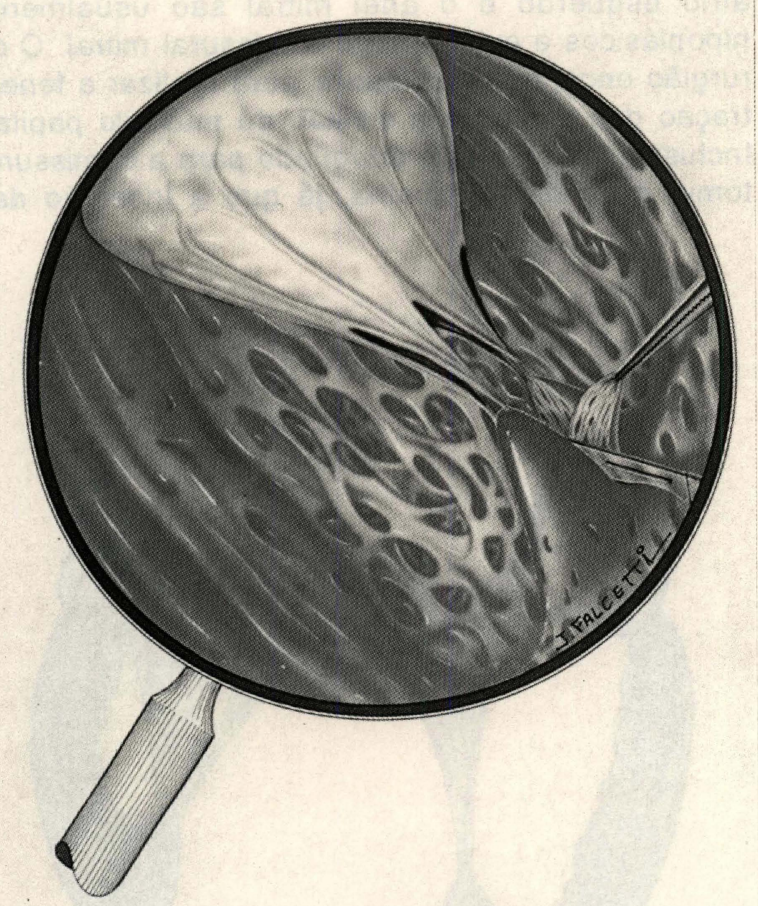

Fig. 1- O músculo papilar único é dividido em dois, lateral e medial. Uma fatia de músculo é ressecada para aumentar a mobilidade.

A seguir, cada um dos "novos" músculos papilares resultantes foi dividido em outros dois, conforme a inserção das cordas, ficando dois destes com orientação mais anterior e dois com orientação mais posterior (Figura 2). Seguindo a orientação determinada pela divisão muscular, as cordas foram sepa-

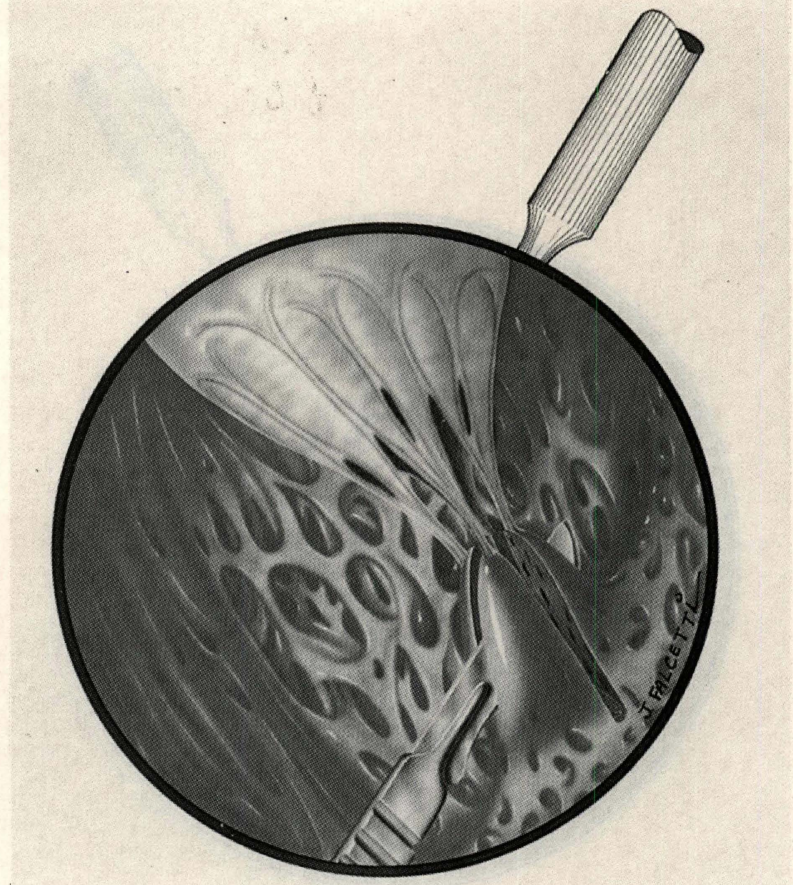

Fig. 2 - Os dois novos músculos papilares são subdivididos conforme a inserção das cordas em duas porções anteriores e duas posteriores.

radas e fenestradas; o excesso de tecido intercordal foi ressecado (Figura 3 ).

Finalmente, as cúspides mitrais foram abordadas, sempre pela via ventricular. As "comissurotomias" foram realizadas em local adequado, acompanhandose a inserção das cúspides até $1 \mathrm{~mm}$ do anel mitral (Figura 3).

Em dois pacientes e ventriculotomia esquerda foi também utilizada para fechar defeitos interventriculares múltiplos, usando um retalho de pericárdio bovino. Em dois pacientes o procedimento de istmosubclavioplastia foi utilizado para correção da coarctação de aorta.

Um paciente apresentava membrana supravalvar mitral, ressecada através de atriotomia esquerda. Em um paciente a estenose fibromuscular subaórtica foi ressecada através de aortotomia. Em um paciente com dupla via de saída do ventrículo direito com comunicação interventricular restritiva e não relacionada a correção total não foi considerada possível, porém, como medidas paliativas, a abertura da valva mitral, a atriosseptectomia e o aumento da comunicação interventricular foram realizados. Somente em um paciente, o tratamento isolado da valva mitral foi feito; esta paciente tinha sido submetida a istmosubclavioplastia um mês antes do tratamento da valva mitral. Neste caso, na primeira operação, o estudo ecocardiográfico pré-operatório 
BARBERO-MARCIAL, M.; RISO, A.; ALBUQUERQUE, A.; ATIK, E.; JATENE, A. D. - A ventriculotomia apical esquerda para tratamento cirúrgico da estenose mitral congênita. Rev. Bras. Cir. Cardiovasc., 6 (3):167-173, 1991.

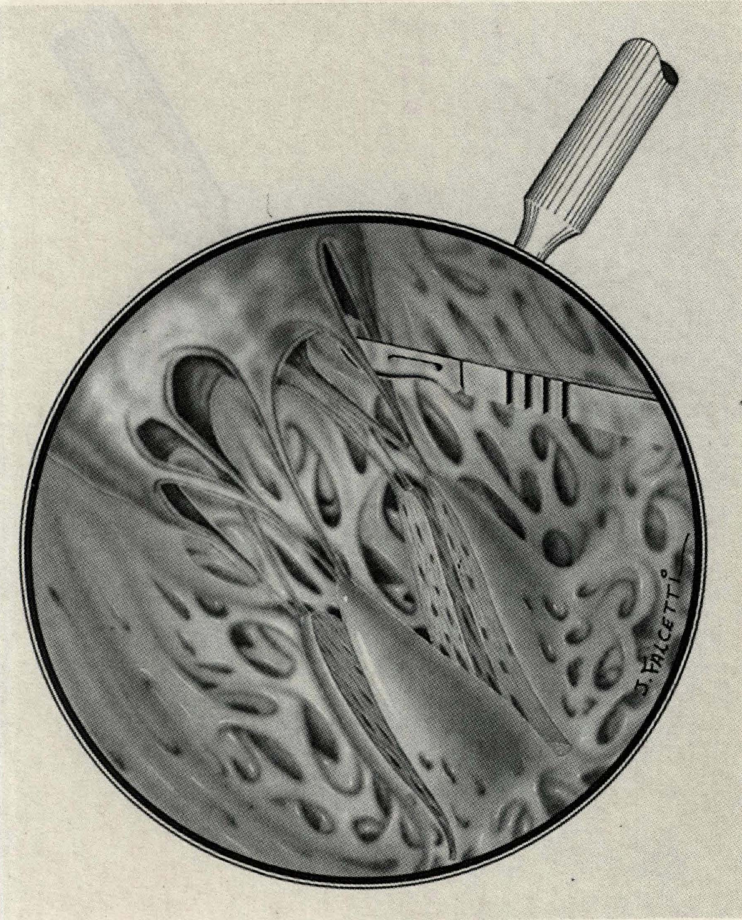

Fig. 3 - Seguindo a orientação determinada pela divisão muscular as cordas são divididas e fenestradas.

não demonstrou valva mitral estenótica em páraquedas. Após a citada correção da coarctação da aorta, teve uma evolução tormentosa pós-operatória, o diagóstico da lesão mitral foi realizado e a sua correção em caráter de emergência.

Finalmente, um paciente apresentou duplo aparelho mitral com anéis mitrais independentes; uma valva mitral era hipoplásica, em posição medial, com anel supravalvar e comunicava o átrio esquerdo com o ventrículo esquerdo, terminando num músculo papilar único. A outra valva em pára-quedas, menos hipoplásica, mas conectava-se com o átrio esquerdo (Figura 4). Uma verdadeira parede atrial separava esta valva da cavidade atrial. Os aparelhos subvalvares foram tratados como previamente descrito, a membrana supravalvar foi ressecada através do átrio esquerdo. Na outra valva, uma pinça foi introduzida retrogradamente através da ventriculotomia; na área correspondente, a parede atrial foi ressecada, resultando, assim, em dois aparelhos mitrais independentes.

\section{RESULTADOS}

Não houve óbitos hospitalares. Um paciente, que se encontrava em calsse funcional $I$, faleceu de causa não relacionada, um ano após a operação. No seguimento pós-operatório, num período variável entre seis e 44 meses, sete pacientes encon- travam-se em classe funcional I e um em II; este último é o de número 6 (Tabela 1), portador de anomalia complexa e submetido a cirurgia paliativa.

O estudo ecocardiográfico seriado, realizado a cada três meses, mostrou adequada velocidade do fluxo através da valva mitral, ausência de gradiente entre átrio esquerdo e ventrículo esquerdo, área da valva mitral acima do normal em sete dos oito pacientes estudados; em um paciente, o de número 6 , a área foi de $1,9 \mathrm{~cm} 2$ (normal $3,0 \mathrm{~cm}^{2}$ ). A regurgitação mitral foi encontrada, em grau mínimo ou moderado, em sete dos oito pacientes (Tabela 2).

A função ventricular esquerda foi considerada dentro dos limites da normalidade, em todos os casos.

\section{COMENTÁRIOS}

A via atrial para abordar a valva mitral em páraquedas encontra limitaçōes, já que a obstrução encontra-se no músculo papilar e nas suas cordas. 0 átrio esquerdo e o anel mitral são usualmente hipoplásicos e existe fusão comissural mitral. O cirurgião encontra dificuldades para realizar a fenestração das cordas e a divisão do músculo papilar. Inclusive, o local mais adequado para a comissurotomia é de difícil escolha, já que a inserção das

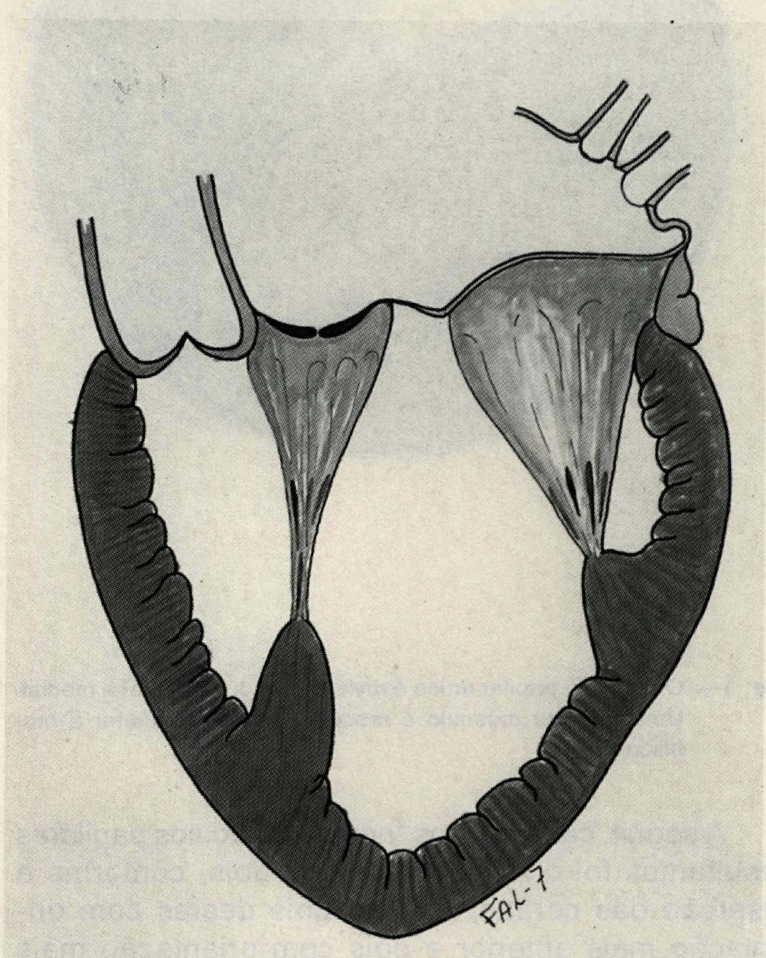

Fig. 4 -Aspecto anatômico do caso de número 7 com dois aparelhos valvares mitrais independentes. 
BARBERO-MARCIAL, M.; RISO, A.; ALBUQUERQUE, A.; ATIK, E.; JATENE, A. D. - A ventriculotomia apical esquerda para tratamento cirúrgico da estenose mitral congênita. Rev. Bras. Cir. Cardiovasc., 6 (3):167-173, 1991.

TABELA 2

DADOS PÓS-OPERATÓRIOS DA ECODOPPLERCARDIOGRAFIA: 6 A 44 MESES DE SEGUIMENTO.

\begin{tabular}{ccccccc}
\hline $\begin{array}{c}\text { Caso } \\
N^{0}\end{array}$ & $\begin{array}{c}\text { Velocidade do } \\
\text { Fluxo Mitral } \\
\text { m./seg }\end{array}$ & $\begin{array}{c}\text { Gradiente } \\
\text { AE/VE }\end{array}$ & $\begin{array}{c}\text { Mitral. P.H.T. } \\
\text { (cm2) }\end{array}$ & $\begin{array}{c}\text { Regurgitação } \\
\text { Mitral }\end{array}$ & \multicolumn{2}{c}{$\begin{array}{c}\text { Função Ventricular } \\
\text { Esquerda }\end{array}$} \\
\hline 1 & 0,96 & Nenhum & 4,6 & Mínima & 0,78 & FS\% \\
2 & 1,02 & Nenhum & 4,2 & Mínima & 0,70 & 33 \\
3 & 1,04 & Nenhum & 4,1 & Moderada & 0,81 & 40 \\
4 & 0,99 & Nenhum & 3,4 & Mínima & 0,80 & 40 \\
5 & 0,97 & Nenhum & 3,3 & Moderada & 0,77 & 38 \\
6 & 0,50 & Nenhum & 1,9 & Nenhuma & 0,63 & 28 \\
7 & 1,30 & Nenhum & 4,4 & Moderada & 0,82 & 41 \\
8 & 1,10 & Nenhum & 4,6 & Moderada & 0,83 & 42 \\
\hline
\end{tabular}

$\mathrm{AE}$ = átrio esquerdo; $\mathrm{VE}$ = ventrículo esquerdo; P.H.T. = "pressure half time”, área valvar mitral calculada por Doppler contínuo; FE = fração de ejeção; $\mathrm{m} . / \mathrm{seg}$. = metros por segundo; $\mathrm{FS}=$ fração segmentar. Velocidade normal de fluxo mitral $=1,0(0,8-1,3) \mathrm{m} . / \mathrm{seg}$. Área mitral normal > $3,0 \mathrm{~cm} 2$.

cordas na face ventricular das cúspides é mal visibilizada. SHONE et alii ${ }^{8}$, posteriormente, outros autores $1,2,4$ relataram a dificuldade de identificar os componentes valvares, mesmo após "dupla comissurotomia"; estas dificuldades têm levado à substituição valvar em um número significante de pacientes.

Levando em consideração que, neste tipo de anomalia mitral, a obstrução é a conseqüência de um músculo papilar único, inserido perto do ápex da parede ventricular livre, nós preconizamos a abordagem direta do aparelho subvalvar através de ventriculotomia apical. 0 músculo é dividido e as cordas divididas, ressecadas, ou fenestradas. Após boa visibilização da inserção das cordas na face ventricular da valva, a comissurotomia é realizada.

A possibilidade de interferência da ventriculotomia apical na função ventricular merece consideração. O trabalho pioneiro de REPOGLE et alii ${ }^{7}$ mostra que este tipo de incisão, também chamada de incisão em "boca de peixe", não provoca alteração na função ventricular esquerda, nem interrup- ção de fonte significante de fluxo coronário. A base anatômica desta conclusão é que o ventrículo esquerdo, no seu ápex, tem espessura de poucos milímetros e, por causa da forma ovóide do ventrículo, a tensão desenvolvida durante a sístole é mínima no ápex.

Por outro lado, outros cirurgiōes ${ }^{3,5}$ publicaram alteração da função ventricular esquerda após ventriculotomia para fechamento de comunicaçöes interventriculares múltiplas. A nosso ver, nesta anomalia existe, já no pré-operatório, importante disfunção ventricular; por outro lado, a sutura de um retalho no septo provoca áreas de isquemia ou, eventualmente, de necrose.

Não série de pacientes apresentada, a avaliação hemodinâmica pós-operatória e os estudos seriados ecocardiográficos pós-operatórios até 44 meses, focalizando, especificamente, a função do ventrículo esquerdo, não demonstraram nenhuma disfunção significante na função ventricular. Obviamente, uma análise pós-operatória mais tardia é necessária para esclarecer, propriamente, o efeito da incisão apical no ventrículo. 
BARBERO-MARCIAL, M.; RISO, A.; ALBUQUERQUE, A.; ATIK, E.; JATENE, A. D. - A ventriculotomia apical esquerda para tratamento cirúrgico da estenose mitral congênita. Rev. Bras. Cir. Cardiovasc., 6 (3):167-173, 1991.

RBCCV 44205-147

BARBERO-MARCIAL, M.; RISO, A.; ALBUQUERQUE, A.; ATIK, E.; JATENE, A.D. - The surgical treatment of congenital mitral stenosis through a left ventriculotomy. Rev. Bras. Cir. Cardiovasc., 6 (3):167173, 1991.

ABSTRACT: From June 1987 to October 1990, nine patients with congenital mitral stenosis (CMS) underwent surgical correction through an apical left ventriculotomy. Seven patients had parachute mitral valve and two had other forms of CMS. The surgical treatment of the mitral valve apparatus starts from below: first the papillary muscle is split and the cordae are divided and fenestrated. Then, the commissurotomies were performed through the ventricles aspect of the mitral valve. Associated anomalies were corrected simultaneously. There was no in-hospital death and only one late death (nonrelated). The echocardiographic showed non significant residual stenosis and normal global function of the left ventricle. In conclusion, the appical left ventriculotomy is a good approach for treatment of CMS.

DESCRIPTORS: heart valves, mitral, surgery; ventriculotomy, apical, left.

\section{REFERÊNCIAS BIBLIOGRÁFICAS}

CARPENTIER, A.; BRANCHINI, B.; COUR, J.C.; VILLANI, M.; DELOCHE, A.; RELLAND, J.; D'ALLAINES, C.; BLONDEAU, P.; PIWNICA, A.; PARENZAN, L.; BROM, G. - Congenital malformations of the mitral valve in children: pathology and surgical treatment. J. Thorac Cardiovasc. Surg., 72: 854-866, 1990.

COLES, J.G.; WILLIAMS, W.G.; WATANABE, T.; DUNCAN, K.F.; SHERRET, H.; DASMAHAPATRA, H.K.; FREEDOM, R.M.; TRUSLER, G.A - Surgical experience with reparative techniques in patients with congenital mitral valvular anomalies. Circulation, 76 (Supl. 3): 117-122, 1987.

KEITH, J.D.; ROWE, R.D.; VLAP, P. - Heart disease in infancy and childhood. New York, MacMillan, 1958, p. 577.

LASKS, H.; HELLENBRAND, W.E.; KLEINMAN, C; TALVER, N.S. - Left atrial ventricle conduit for relief of congenital mitral stenosis in infancy. J. Thorac. Cardiovasc. Surg., 80: 782-787, 1980.

MITCHELL, I.M.; PRABHAKAR, G.; MAUGHAN, J.; TAYLOR, D.N. - Pulmonary artery versus left ventricular venting: a radioisotope study of left ventricular function. Ann. Thorac. Surg., 48: 699-703, 1989.

NADAS, A.S. \& FYLER, D.C. - Pediatric cardiology. 3. ed. Philadelphia, W.B. Saunders, 1972. p. 683-687.

REPOGLE R.L.; KUNDLER H.; GROSS, R.E. - Left vintricular performance following apical left ventriculotomy. Arch. Surg., 95: 892-295, 1969.

SHONE, J.D.; SELLERS, R.D.; ANDERSON, R.C.; ADAMS Jr., P.; LILLEHEI, C.W.; EDWARDS, J.E. - The developmental complex of "parachute mitral valve", supravalvular ring of left atrium, subaortic stenosis and coarctation of aorta. Am. J. Cardiol., 11: 714725, 1963.

9 TAGUCHI, K. \& NAKAMURA, S. - Congenital mitral stenosis: report of three surgical cases and a review of the literature. Hiroshima J. Med. Sci., 16: 267$270,1967$.

\section{Discussão}

\section{DR. DÉCIO OLIVEIRA ELIAS Maceió, $A L$}

A estenose mitral congênita é uma lesão de difícil manuseio cirúrgico, pela variedade de componentes do aparelho mitral geralmente envolvida, anel, cúspides, cordoalha e músculos papilares, em associaçōes as mais diversas. Em mais de $60 \%$ dos casos, está associada a outras lesōes, principalmente a comunicação interventrícular, coarctação da aorta e dupla via de saída de ventrículo direto, o que também encontramos em nossa experiência. Podemos identificar na estenose mitral congênita: hipoplasia do anel mitral, espessamento das cúspides, ausência de comissuras, encurtamento ou fusão de cordas com obliteração fibrosa intercordal, anomalias de inserção das cordas, hipoplasia de músculos papilares, fusão dos papilares, músculo papilar único, músculos papilares múltiplos, e membrana fibrosa supravalvar. Carpentier simplificou a análise da estenose mitral congênita, classificando-a em dois tipos principais, conforme a presença ou a ausência de anomalias dos músculos papilares. Tipo A - músculos papilares normais: 1) fusão comissuro-papilar; 2) tecido valvar excessivo; 3) hipoplasia anular; 4) 
BARBERO-MARCIAL, M.; RISO, A.; ALBUQUERQUE, A.; ATIK, E.; JATENE, A. D. - A ventriculotomia apical esquerda para tratamento cirúrgico da estenose mitral congênita. Rev. Bras. Cir. Cardiovasc., 6 (3):167-173, 1991.

anel supravalvar. Tipo B - músculos papilares anormais: 1) músculo papilar (V. pára-quedas); 2) músculos papilares múltiplos (V. rede de dormir); 3 ) músculos papilares ausentes. No tipo $A$, em que os músculos papilares são normais, as alteraçōes consistem em fusão das comissuras aos papilares, com ausência de cordas, excesso de tecido das cúspides, hipoplasia do anel mitral e a presença de um anel fibroso supravalvar que imobiliza as cúspides. 0 grupo $B$ inclui a presença de um único papilar onde se inserem todas as cordas, livres ou fusionadas, e que caracteriza a "valva em pára-quedas", forma mais comum do grupo e encontrada em oito dos nove casos estudados pelos autores. Inclui, ainda, a existência de papilares múltiplos com inserção desordenada das cordas (valva em rede de dormir) $e$, finalmente, a ausência de papilares em que as cordas se inserem na parede ventricular. Para o tratamento da EMC dispomos de três métodos: 1) procedimentos reconstrutivos (plastias); 2) substituição valvar; 3) conduto valvado atrioventricular esquerdo. Procedimentos reconstrutivos, em que se busca ampliar a área valvar e, principalmente, a região subvalvar, criando comissuras, isolando cordas fusionadas ou dividindo o papilar único. Esses procedimentos devem ser adequadamente balanceados, de modo a resultar no menor gráu possível de insuficiência mitral, o que foi conseguido com grande sucesso nos casos estudados pelos autores. Substituição da valva mitral. Indicada quando os procedimentos reconstrutivos não são aplicáveis ou quando a insuficiência mitral resultante da plastia é inaceitável. Em nossa pequena experiência de quatro casos, pudemos ressecar uma membrana fibrosa supravalvar e ampliar comissuras em apenas um caso, sendo os três restantes submetidos a troca da valva, dois dos quais por insuficiência mitral severa pós plastia, sem sucesso, e que foram a óbito por baixo débito cardíaco, no pós-operatório imediato. Quando o elemento obstrutivo mais importante é o anel mitral, com severa hipoplasia, a solução, via de regra, inclui o "bypass" da valva mitral por um conduto valvado extracardíaco, unindo o átrito e ventrículo esquerdos. Corno e colaborado- res, em 1986, descreveram um caso de hipoplasia mitral, que trataram com um conduto valvado atriventricular esquerdo. Através da ventriculotomia apical esquerda, puderam visibilizar e examinar o papilar único da valva em pára-quedas hipoplásica. A adoção da ventriculotomia apical esquerda, como preconizada pelos autores para o manuseio dessa complexa lesão, sem dúvida, permite uma análise muito mais minuciosa e informativa do aparelho subvalvar, quando comparado com o acesso atrial convencional. O planejamento e a execução do tratamento reparador, como vimos durante a excelente apresentação, ficam extraordinariamente simplificados e permitindo aos autores a obtenção de resultados que não são igualados por outros métodos de tratamento. Agradecemos ao Dr. Miguel Barbero-Marcial e colaboradores ter partilhado conosco essa sua excelente e criativa experiência que, acreditamos, tornará a ventriculotomia apical esquerda a via de eleição para tratamento da estenose mitral congênita nas formas em que há anomalias das estruturas subvalvares.

Finalizando, gostaria de cumprimentar os autores, pela excelência dos resultados obtidos com - tratamento dessa difícil lesão e perguntar ao Dr. Miguel se, durante o período do estudo, outros pacientes com estenose mitral congênita necessitaram das outras formas de tratamento, como a substituição valvar, ou o conduto atrioventricular esquerdo.

\section{DR. BARBERO-MARCIAL (Encerrando)}

Agradeço os comentários do Sr. Décio, que, de maneira precisa, nos apresentou todos os problemas e dificuldades que o cirurgião encontra, ao se propor tratar a estenose mitral congênita. Respondendo à sua pergunta, gostaria de dizer que os casos apresentados foram consecutivos e nenhum outro paciente portador dessa lesão foi operado por outra técnica. Mais uma vez, muito obrigado. 